\title{
Partizipative Planung mit Seniorinnen und Senioren am Beispiel von Zukunftswerkstätten in Pirmasens
}

\author{
Pia Gerhards · Annette Spellerberg
}

Eingegangen: 17. August 2010 / Angenommen: 8. März 2011 / Online publiziert: 30. März 2011

(C) Springer-Verlag 2011

\begin{abstract}
Zusammenfassung Die wachsende Zahl älterer Menschen in den Kommunen führt zu der Notwendigkeit, die Planung für diese Zielgruppe anzupassen und so die Voraussetzungen zur Erhaltung von Lebensqualität zu schaffen. Um die Planungen für ältere Menschen in der rheinland-pfälzischen Stadt Pirmasens zu unterstützen, wurden im Rahmen eines Projektes neben einer repräsentativen Seniorenbefragung und Experteninterviews sechs Zukunftswerkstätten in unterschiedlichen Stadtteilen durchgeführt. In diesem Aufsatz wird aufgezeigt, inwieweit Zukunftswerkstätten ein geeignetes Instrument darstellen, um einerseits Wünsche und Bedürfnisse zu ermitteln und andererseits ältere Menschen anzuregen, sich selbst für die Umsetzung ihrer geäußerten Anliegen zu engagieren.
\end{abstract}

Schlagwörter Wohnen älterer Menschen ·

Zukunftswerkstatt · Planung für Senioren ·

Bürgerbeteiligung

\section{Participatory Planning with Elderly People-The Example of "Zukunftswerkstätten"}
Abstract The increasing number of elderly people neces- sitates enhancing the local planning of municipalities for this target group and creating adequate conditions for the preservation of quality of life. As part of a project concern- ing planning for elderly people in the Rhineland-Palatinate
P. Gerhards $(\varangle) \cdot$ Prof. Dr. A. Spellerberg
Lehrgebiet Stadtsoziologie, Technische Universität Kaiserslautern, Pfaffenbergstraße 95, 67663 Kaiserslautern, Deutschland
E-Mail: pia.gerhards@rhrk.uni-kl.de
Prof. Dr. A. Spellerberg
E-Mail: spellerb@rhrk.uni-kl.de

city of Pirmasens, besides a representative survey and interviews with experts, the participative process "Zukunftswerkstatt", a form of creative workshops, was accomplished in six different districts. This article points out, to what extent the creative workshops represent a suitable instrument to identify the desires and needs of the elderly and if these workshops can activate the older people to engage in the realization of their expressed needs.

Keywords Housing of elderly people $\cdot$ Creative workshops · Planning for the elderly · Public participation

\section{Einleitung: Notwendigkeit des Einbezugs Älterer in die Planung}

Als „Senioren“, „ältere Menschen“ oder einfach „Ältere“ werden Personen bezeichnet, die ein gewisses Lebensalter erreicht oder überschritten haben. Häufig wird das biologische Lebensalter, und zwar das 60. oder 65. Lebensjahr, als Abgrenzung verwendet. Diese Merkmale werden gewählt, weil das Ausscheiden aus dem Erwerbsleben bereits stattgefunden hat oder kurz bevor steht. Allerdings ist es häufig zielführender, die Lebensphase „Alter“ in Kategorien der Selbstständigkeit einzuteilen, von einer durch Aktivität geprägten bis hin zu einer von anderen Menschen abhängigen Lebenssituation. Der Wiener Soziologe Leopold Rosenmayr etwa unterteilt das Alter in eine chancenreiche Phase, in der die Fähigkeit der Leistungserbringung für andere Menschen noch besteht, eine eingeschränkte Phase, in der Kompetenzen abnehmen und schließlich eine von Hilfe und Pflege abhängige Phase (Rosenmayr 1996: 35). Einhergehend mit dem Alterungsprozess treten körperliche Einschränkungen und gesundheitliche Probleme häufiger auf, so dass der Hilfebedarf tendenziell zunimmt. Innerhalb der Gruppe der 
„Älteren“ bestehen aber große individuelle Unterschiede, etwa hinsichtlich der körperlichen und geistigen Leistungsfähigkeit, aber auch der Interessen und Neigungen und der persönlichen Lebenssituation. Ältere Menschen sind heute häufig sehr mobil, gestalten ihr Leben selbstständig und verfügen über einen guten Gesundheitszustand. Auch Einstellungen und Lebensstile haben sich gewandelt. Viele ältere Menschen wollen aktiv am Leben in der Gemeinschaft teilhaben und es mitgestalten (vgl. Heetderks/Nell 2008: 22). Erst bei stärkeren gesundheitlichen Beeinträchtigungen treten sie von ihren Freizeitaktivitäten zurück.

Eine Reihe von Studien belegt, dass viele Ältere den Großteil ihrer Zeit in ihrer Wohnung oder der unmittelbaren Wohnumgebung verbringen (vgl. z. B. Friedrichs 1990: 175; Thabe 1997: 8; Engstler/Menning/Hoffmann et al. 2004: 236). Wohnbedingungen und die Qualität des Umfelds sind daher von gesteigerter Bedeutung für diese Gruppe. Zugleich wird immer wieder darauf hingewiesen, dass ältere Menschen so lange wie möglich selbstständig und selbstbestimmt in ihren vertrauten Wohnungen wohnen bleiben möchten (vgl. z. B. Höpflinger 2004: 93; Banse/ Möbius/Deilmann 2008: 42). Der Verwirklichung dieses Wunsches steht allerdings oft eine Reihe von Hürden im Wege, die in der Gestaltung und Ausstattung von Wohnung und Wohnumfeld bestehen.

Das hier beschriebene Projekt (vgl. Spellerberg/Krickel 2010) orientiert sich vor allem an der Gestaltung des öffentlichen Raums und den Angeboten in den Wohngebieten. Um die Bedürfnisse der älteren Menschen in ihren jeweiligen Wohnlagen genauer zu erfassen und sie gleichzeitig an der Planung und Umsetzung von Maßnahmen zu beteiligen, wurde die Methode der Zukunftswerkstätten eingesetzt. Die Senioren werden dabei selbst als Experten für ihre eigene Lage betrachtet. Sie kennen sich vor Ort aus und wissen, woran es fehlt und welchen Anforderungen die Wohngebiete nicht gerecht werden. Deshalb ist es von großer Bedeutung für die bedürfnisgerechte Umgestaltung der Wohnquartiere, diese „Experten in eigener Sache“ einzubeziehen. Durch die Offenheit und gleichzeitig Zielgerichtetheit des Verfahrens bietet die Zukunftswerkstatt besondere Vorteile, denn sie fördert gegenüber anderen Beteiligungsverfahren (etwa „Open Space“ oder Planungszelle; vgl. dazu Bischoff/Selle/ Sinning 2007) sehr schnell die zentralen Informationen zutage und führt so in kurzer Zeit zu umsetzbaren Ergebnissen. Für ältere Menschen ist der zeitliche Faktor von besonderer Bedeutung. Darüber hinaus ist ein Ziel der Studie, die älteren Menschen zu aktivieren und ihnen die Möglichkeit zu geben, an der Planung der Kommune in den sie betreffenden Bereichen zu partizipieren.

Das Verfahren der Zukunftswerkstatt knüpft auch an die aktuelle Diskussion um das freiwillige oder bürgerschaftliche Engagement Älterer an, das in den letzten Jahren verstärkt Aufmerksamkeit gewonnen hat (vgl. dazu etwa
Karl/Aner/Bettmer et al. 2008 und die Sammelbände von Schroeter/Zängl 2006 und Erlinghagen/Hank 2008). Viele Ältere verfügen über Kenntnisse, Fähigkeiten und zeitliche Ressourcen, die der Gesellschaft nutzen können (vgl. z. B. Braun/Bischoff 1999: 67 ff.; Deutscher Bundestag 2002: 101; Schmidt 2004; Bundesministerium für Familie, Senioren, Frauen und Jugend 2010: 19). Die Defizitorientierung, die lange Zeit die Diskussion um das Alter(n) beherrschte, scheint überholt. Das Bild, das Senioren einseitig als gebrechlich, krank und hilfebedürftig beschreibt, ist nicht mehr angemessen (vgl. Brauers 2008: 215). Stattdessen werden die Potenziale und Chancen mehr ins Blickfeld gerückt. Derzeit stehen Konzepte des ,aktiven“ oder „,produktiven“ Alterns im Vordergrund. Dabei wird die Nützlichkeit der älteren Menschen für sich selbst, andere und die Gesellschaft betont (vgl. Petermann/Roth 2006: 248 f.). Gestützt werden diese Annahmen auch durch empirische Studien, wie etwa den „Freiwilligensurvey“, nach dem insbesondere das Engagement der ,jungen Alten“"zwischen 60 und 69 Jahre zwischen 1999 und 2009 stark zugenommen hat (vgl. Gensicke 2010: 229 f.).

Durch die Beteiligung und den aktiven Einbezug der Älteren in die Planung erhalten sie eine Möglichkeit, sich für ihr Umfeld und die Gesellschaft zu engagieren. Die direkte Betroffenheit, die dadurch gegeben ist, dass das Ziel des Engagements die eigene unmittelbare Wohnumgebung ist, wirkt dabei besonders motivierend und erhöht die Bereitschaft, über eine einmalige Veranstaltung hinaus an der Problemlösung mitzuwirken (vgl. Selle 1996: 78). Das freiwillige Engagement bietet aber nicht nur Vorteile für die Allgemeinheit, sondern wirkt sich auch positiv für die Engagierten selbst aus. Sie erhalten soziale Anerkennung und erfahren Gemeinschaft, was das Wohlbefinden steigert und mit einer besseren Gesundheit einhergeht (vgl. Haski-Leventhal 2009: 398). Gleichzeitig wird den Bürgern zunehmend mehr Verantwortung zugestanden, Selbsthilfe und der persönliche Einsatz des Einzelnen werden verstärkt auch von Seiten der Politik gefordert. Dies verpflichtet auch die Kommunen, die Möglichkeiten des Engagements und der aktiven sowie aktivierenden Beteiligung der Betroffenen zu fördern (Rohden/Villard 2010: 55).

Es stellt sich daher die Frage, wie Zukunftswerkstätten gewährleisten können, Wünsche und Bedürfnisse älterer Menschen offenzulegen und Ältere anzuregen, sich an der Verwirklichung dieser Wünsche zu beteiligen. Zu diskutieren ist weiterhin, wie gewährleistet werden kann, dass auch die anschließende Umsetzung erfolgreich verläuft.

\section{Datengrundlage und Aufbau der Gesamtstudie}

Die kreisfreie Stadt Pirmasens ist ein Mittelzentrum in der Region Südwestpfalz mit rund 41.000 Einwohnern (Stand 
2009; 48.000 Einwohner 1987) und liegt westlich des Pfälzerwaldes. Einige Stadtteile sind ehemals eigenständige Vororte, die einen eher dörflichen Charakter haben. Pirmasens ist mit einem Anteil von Senioren (Personen über 60 Jahre) an der Gesamtbevölkerung von rund $30 \%$ die derzeit ,älteste' Stadt in Rheinland-Pfalz, was durch den starken Bevölkerungsrückgang vor allem bei Jüngeren begründet ist. Zukünftig wird sich der Anteil Älterer in Pirmasens, wie auch in vielen anderen Städten und Regionen Deutschlands, noch erhöhen. In Rheinland-Pfalz wird nach Daten des Bundesinstituts für Bau-, Stadt- und Raumforschung (BBSR) (2010) der Anteil der Personen ab 60 Jahre 2025 bei $32 \%$ liegen, bei einem Bevölkerungswachstum von $1 \%$. In Pirmasens liegt die Quote der Über-60-Jährigen 2025 bei $36 \%$, bei einem Fortbestehen der Schrumpfung von $10 \%$. Die geringere Zunahme des Anteils der Älteren (um etwa $7 \%$ im Vergleich zu $30 \%$ in Rheinland-Pfalz) ist darauf zurückzuführen, dass die Bevölkerung bereits heute sehr stark gealtert ist. ${ }^{1}$

Die aktuelle Situation in Pirmasens ist - neben den deutschlandweit auftretenden Trends der negativen natürlichen Bevölkerungsentwicklung und der Suburbanisierung - eine Folge der wirtschaftlich schwierigen Lage vor Ort. Als ehemalige Schuhmetropole ging es der Stadt bis in die 1970er Jahre wirtschaftlich sehr gut. Mit der zunehmenden wirtschaftlichen Globalisierung wurde der Stadt seit den 1980er Jahren aber mehr und mehr die Grundlage ihrer Industrie genommen. Es kam zu einem nachhaltigen Verlust von Arbeitsplätzen und einer anhaltend starken Abwanderung junger und mobiler Bevölkerungsgruppen. Der Abzug des amerikanischen Militärs in den 1990er Jahren verschlechterte die Arbeitsmarktsituation erneut und führte in der Summe zu einem andauernden negativen Wanderungssaldo der jüngeren Bevölkerung.

Vor diesem Hintergrund initiierte die Stadt ein Projekt zur zukunftsfähigen Neugestaltung der Planung für ältere Menschen. Ziel war es, die Lebenssituation und -bedingungen älterer Menschen in der Stadt sowie ihre Wünsche und Problemwahrnehmungen zu analysieren und damit eine bedürfnisgerechte Planung für diese Zielgruppe zu unterstützen. Im Vordergrund stand dabei, die Lebensqualität der Senioren zu erhalten und zu erhöhen sowie die Nutzung der sich daraus ergebenden Chancen für die Gesellschaft, etwa durch ehrenamtliches Engagement, zu verbessern.

Dabei kam ein Methodenmix mit drei Erhebungsschritten zum Einsatz. Zunächst wurde eine repräsentative, postalische Befragung von älteren Bürgern durchgeführt, parallel dazu fanden Experteninterviews mit Vertretern

\footnotetext{
${ }^{1}$ Für Deutschland geht die 12. koordinierte Bevölkerungsvorausberechnung von einem Anstieg des Anteils der 60-Jährigen und Älteren von $19 \%$ im Jahr 2005 auf zwischen 36 und $37 \%$ im Jahr 2030 aus (Statistisches Bundesamt 2009: 39 ff.). Gleichzeitig wird die Gesamtbevölkerung um 2 bis 6 Prozentpunkte zurückgehen.
}

seniorenrelevanter Einrichtungen und Diensten statt. Auf diese Weise bestand bereits ein Einblick in die Sichtweise der Zielgruppen und unterschiedlichen Akteure, bevor in einem dritten Schritt die Zukunftswerkstätten veranstaltet wurden, um die älteren Menschen in den Prozess mit einzubeziehen und dabei unterschiedliche Wohnlagen in der Stadt zu berücksichtigen.

Der Anstoß zu dem Projekt erfolgte auf Initiative der Stadtverwaltung Pirmasens. Von Anfang an war es ein wichtiges Anliegen der Stadt, die Betroffenen selbst einzubeziehen. Es wurde deutlich gemacht, dass das Konzept nur gelingen könne, wenn die Personen, für die geplant werden sollte, die Möglichkeit erhalten würden, sich aktiv zu beteiligen und ihre Wünsche mitzuteilen. Die Stadtverwaltung war mit mehreren Mitarbeitern in allen Phasen des Projektes eingebunden, ebenso der Seniorenbeirat und das Seniorenbüro der Stadt Pirmasens. Auch der Bürgermeister unterstützte das Projekt im gesamten Verlauf, was sich auch deshalb als wertvoll und wichtig erwies, da das Projekt so in der Öffentlichkeit stärker wahrgenommen wurde. Die einzelnen Arbeitsschritte wurden mit den verschiedenen Vertretern der Stadt abgestimmt und im Stadtrat mehrfach zur Diskussion gestellt. Eine Diskussion mit Bürgern über Inhalte und Ergebnisse wurde durch zwei Bürgerversammlungen nach der Hälfte der Projektlaufzeit und zum Abschluss möglich. Auch die Berichterstattung in der Presse leistete einen wichtigen Beitrag zur Aktivierung der Bevölkerung.

\subsection{Teilstandardisierte quantitative Erhebung: Leben und Wohnen in Pirmasens}

In der repräsentativen, postalischen Befragung gaben knapp 2000 Senioren Auskunft über ihre Lebenssituation und Problemsicht. Ein Fragebogen wurde an 5000 Personen ab 60 Jahre versandt, die zum Befragungszeitpunkt selbstständig in einer Wohnung bzw. einem Einfamilienhaus wohnten. Die Rücklaufquote betrug $40 \%$.

Die Befragten sind im Durchschnitt 73 Jahre alt und verfügen über ein relativ hohes Einkommen von durchschnittlich $1060 €$ pro Person. Es liegt um $220 €$ höher als bei der Vergleichsgruppe aus dem ALLBUS 2008 (Westdeutsche ab 60 Jahre in Orten unter 50.000 Einwohnern). Die finanzielle Lage der älteren Generation stellt sich somit gegenüber der Gesamtsituation der Pirmasenser Einwohner vergleichsweise positiv dar und wird auf die frühere Verfügbarkeit von Arbeitsplätzen in der Schuhindustrie für Männer und Frauen zurückgeführt. $64 \%$ der Befragten leben in 2-Personen-Haushalten, davon die überwiegende Mehrheit von $94 \%$ mit ihrem (Ehe-)Partner. $30 \%$ aller Befragten leben allein. Von den Personen in 2- und Mehrpersonenhaushalten leben insgesamt $12 \%$ mit ihren Kindern.

\footnotetext{
${ }^{2}$ Allgemeine Bevölkerungsumfrage der Sozialwissenschaften.
} 
Das selbstbestimmte Wohnen ist ein zentrales Anliegen der Befragten. Der Großteil der älteren Menschen in Pirmasens $(75 \%)$ möchte auch im hohen Alter die aktuelle Wohnsituation aufrechterhalten. Die Wohnumgebung ist allerdings nach Meinung der Befragten häufig nicht für eine Nutzung im hohen Alter gestaltet. Insbesondere Begegnungsstätten, Freizeitmöglichkeiten und Poststellen sind für viele nicht in der unmittelbaren Wohnumgebung erreichbar (vgl. Abb. 1). Die Vororte sind verstärkt betroffen, aber auch in der Innenstadt besteht dieses Problem. Die Erreichbarkeit einer Bushaltestelle ist für fast alle Befragten gegeben, was die Mobilität Älterer prinzipiell begünstigt, jedoch nutzen nur knapp ein Drittel der Befragten die öffentlichen Verkehrsmittel.

Dennoch äußern viele der befragten älteren Menschen Zufriedenheit mit ihren Wohnumgebungen. Insbesondere die Beziehungen zu Nachbarn werden von vielen positiv bewertet $(91 \%$ sind im Hinblick darauf sehr oder eher zufrieden). Höhere Unzufriedenheit besteht dagegen mit den für ältere Menschen wichtigen Sitzbänken. Fast die Hälfte gibt an, damit sehr oder eher unzufrieden zu sein. Auch die Sauberkeit der Straßen und Gehwege wird von $38 \%$ schlecht bewertet. Die Versorgungssituation in Bezug auf Lebensmittel ist für ein Viertel der Befragten nicht zufriedenstellend. Dabei weisen insbesondere Personen mit gesundheitlichen Einschränkungen höhere Unzufriedenheitswerte auf.

Die Befragungsergebnisse weisen außerdem auf ein hohes Potenzial in Bezug auf das freiwillige Engagement unter der älteren Bevölkerung hin. $36 \%$ geben an, bereits freiwillig oder ehrenamtlich tätig zu sein. Darüber hinaus besteht zusätzliches Potenzial bei Personen, die bisher nicht freiwillig engagiert sind: Weitere $6 \%$ (127 Personen) äußern Interesse an der Übernahme freiwilliger Tätigkeiten - es fehlt ihnen aber häufig an Informationen oder Anleitung, wo und wie sie sich sinnvoll einsetzen können.

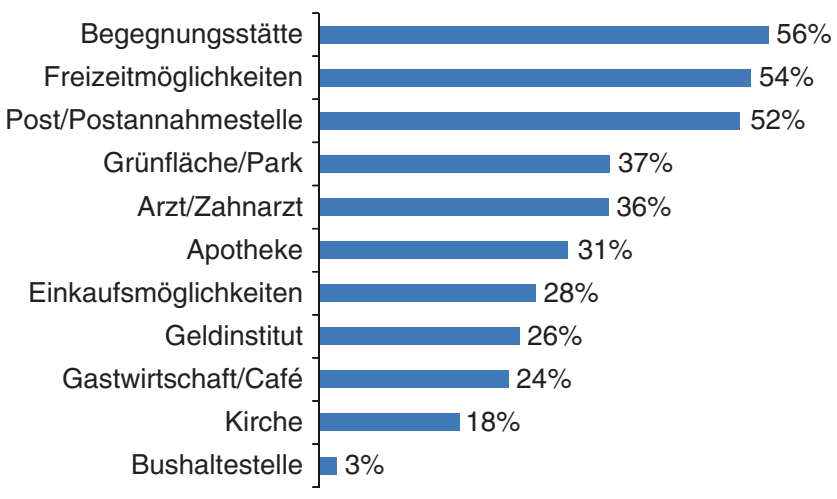

Abb. 1 Für Senioren nicht erreichbare Einrichtungen in der Wohnumgebung. (Quelle: Eigene Erhebung: Seniorenbefragung in Pirmasens $2009, \mathrm{~N}=1224$ bis 1864 )

\subsection{Leitfadengestützte Interviews: Experten vor Ort}

Befragt wurden 21 Experten, unter anderem verantwortliche Personen aus Seniorentreffs (kirchlich und städtisch), Pflegeeinrichtungen, Beratungsstellen, Sportvereinen und pflegeergänzenden Angeboten, wie „Essen auf Rädern“ und Hausnotrufdiensten. Sie beschreiben eine generell gute Ausstattung und Versorgung für Senioren, insbesondere in den Bereichen Freizeit und Beratung, sowie eine überwiegende Zufriedenheit der älteren Menschen. Doch auch aus der Sicht der Experten gibt es Problembereiche, wie etwa mangelnde Barrierefreiheit in den Wohngebieten und in der Folge daraus die Einschränkung der Mobilität älterer Menschen. Auch von den Experten werden fehlende nahräumliche Einkaufsmöglichkeiten angesprochen und als Reaktion darauf Ausbau von Fahr- und Begleitdiensten gewünscht. Gleichzeitig wird bei diesem Aspekt angemerkt, dass auch gegenseitige Hilfe und Unterstützung unter den Senioren vorhanden sei. Defizite sehen einige Experten zudem im Bereich spezieller Pflegeangebote (z. B. Tages- und Nachtpflege) und im Hinblick auf den Einbezug älterer Migranten in das bestehende Angebot, etwa bei Beratung oder Freizeitmöglichkeiten. Die Stärkung und Förderung ehrenamtlicher und freiwilliger Tätigkeiten wird von den Experten als bedeutsamer Faktor benannt. Insbesondere im Bereich der Begleitung und bei Besuchsdiensten bestehe größerer Bedarf an Freiwilligen. Aber sie sehen auch ungenutzte Potenziale für freiwilliges Engagement bei der älteren Generation.

Die Sichtweise der Experten ist, entsprechend des jeweiligen Aufgabengebietes, eher an Problemgruppen orientiert. Sie berichten beispielsweise häufig von bestehenden Schwierigkeiten bei der Ausgestaltung des öffentlichen Nahverkehrs. Darüber hinaus sprechen sie fehlende barrierefreie Zugänge zu öffentlichen Einrichtungen an. Diese Aspekte hatten bei der quantitativen Erhebung nur nachgeordnete Bedeutung. In vielen Bereichen aber stimmt die Wahrnehmung in beiden Erhebungen überein.

\subsection{Zukunftswerkstätten: Senioren gestalten mit}

Im Oktober und November 2009 wurden sechs Zukunftswerkstätten durchgeführt. Hier wurden die nahräumlichen Situationen genauer nach Wohnlage betrachtet. Die insgesamt 18 Stadtteile und sieben eingemeindeten Vororte wurden in sechs Stadtgebiete zusammengefasst. In jedem dieser Gebiete wurde eine Zukunftswerkstatt durchgeführt. Es handelte sich um für alle älteren Bürger öffentliche Veranstaltungen, die zuvor in der örtlichen Presse (Zeitungen und Radio) sowie im Informationsprogramm des Seniorenbüros angekündigt worden waren. Auf eine vorherige Auswahl von Teilnehmern wurde verzichtet. Eine repräsentative Auswahl von infrage kommenden Personen erschien nicht 
sinnvoll, da sonst vielen Interessierten die Teilnahme verwehrt gewesen wäre.

Im Folgenden wird kurz auf die Methode und ihre Bedeutung eingegangen, bevor der Ablauf und die Ergebnisse der Zukunftswerkstätten im Projekt in Pirmasens genauer beschrieben und bewertet werden.

\subsubsection{Methode Zukunftswerkstatt}

Bei Zukunftswerkstätten handelt es sich um eine informelle Form der Kommunikation mit Bürgern, bei der diese zusammengeführt werden, um eigene Ideen und Wünsche für die Lösung drängender Probleme - in diesem Fall die Planung von lebenswerten Stadtteilen für ältere Menschen - einzubringen und zu diskutieren. Das Konzept der Zukunftswerkstatt wurde in den 1960er Jahren von Robert Jungk entwickelt, um Bürger in Prozesse der Entscheidungsfindung einzubeziehen und so Demokratie zu stärken (vgl. Jungk/Müllert 1997: 12 f.). Die Gestaltung der Lebensbedingungen sollte nicht mehr nur den Experten aus Politik und Wirtschaft überlassen werden, sondern die Betroffenen selbst einbeziehen. Die Methode wurde seitdem weiterentwickelt, so dass Zukunftswerkstätten heute in vielen Bereichen in unterschiedlichen Formen und zu unterschiedlichen Themen durchgeführt werden (zum Prozess der Verbreitung des Konzeptes vgl. Müllert 2009). Sie dauern zwischen mehreren Tagen und wenigen Stunden (vgl. Kuhnt/Müllert 2006: 29 ff. und 38; Bischoff/Selle/Sinning 2007: 139). Der Prozess wird von unabhängigen Moderatoren begleitet, die als Initiatoren und Vermittler auftreten. Gemeinsam ist allen Zukunftswerkstätten, dass sie sich in drei Phasen gliedern:

- „Bestandsaufnahme/Kritik“, in der Kritik geübt wird,

- „Phantasie/Utopie“, wo Wünsche zusammengetragen werden und

- „Verwirklichung/Umsetzung“, in der konkrete Projektvorschläge erarbeitet werden.

In einem kreativen Prozess sammeln die Teilnehmer Ideen zur Lösung von Problemen, entwickeln Perspektiven für die Zukunft und planen erste Schritte zur Erreichung der Ziele. Alle Beteiligten sollen möglichst gleichberechtigt zusammenarbeiten, wobei die Themen und die Ergebnisse bewusst offen gehalten werden. Die Teilnehmer bestimmen selbst die relevanten Themenfelder und entwickeln Lösungswege und -ansätze, die ihren Bedürfnissen entsprechen. Ziel ist immer, die Betroffenen selbst bei der Lösung der Probleme einzubeziehen, denn die teilnehmenden Personen sollen angeregt werden, über die einmalige Beteiligung im Rahmen der Veranstaltung hinaus an der Umsetzung der eigenen Ideen mitzuwirken. Es sollen ihnen dazu auch Möglichkeiten und Wege aufgezeigt werden, wie sie dies tun können (vgl. Kuhnt/Müllert 2006: 14 f.; Bischoff/Selle/Sinning 2007: 138 f.). Somit wird mit dieser Methode auch „Hilfe zur Selbsthilfe“ geleistet. Zukunftswerkstätten und die sich daran anschließenden Projekte können also als zeitweiliges „projektbezogenes Engagement in losem institutionellem Rahmen auf Basis freiwilliger Bereitschaft" (Pfenning/ Benighaus 2008: 197) angesehen werden, das sich von traditionellen Formen der Partizipation wie Engagement in Parteien oder Bürgerinitiativen unterscheidet.

Durch das Vorgehen in drei Phasen wird bei zwei Schwierigkeiten angesetzt, die ansonsten in vielen Diskussionen auftreten: Einerseits wird Ordnung in die Vielzahl unterschiedlicher und oft gleichzeitig vorgebrachter Kritikpunkte, Ideen, Hinweise auf Sachzwänge und Zuständigkeiten, Lösungsvorschläge etc. gebracht. Andererseits werden all diese Äußerungen schriftlich und für alle gut sichtbar festgehalten, was verhindert, dass genannte Vorschläge und Anmerkungen ignoriert werden (vgl. Sellnow 1998: 146). Vor allem die „Phantasie/Utopiephase“ hat einen besonderen Stellenwert. Hier können sich die Gedanken und Überlegungen von der Realität lösen, es wird jenseits aller Sachzwänge, Kosten und Zuständigkeiten nach Wünschen und Lösungen gesucht. So kommen oftmals völlig neue Ideen zustande, die anschließend in konkrete Projekte münden.

\subsubsection{Aufbau und Ablauf der Zukunftswerkstätten in Pirmasens}

Die große Einsatzbereitschaft, die schon in der zuvor durchgeführten Seniorenbefragung festgestellt wurde, zeigte sich auch bei der Teilnahme an den Zukunftswerkstätten. Die Veranstaltungen waren gut besucht (insgesamt nahmen 150 Personen teil, davon waren 37 \% Männer) und die Teilnehmer waren durchweg engagiert und interessiert. Es machten sich dennoch Unterschiede in den Teilnehmerzahlen bemerkbar: Die Veranstaltungen in den Vororten hatten einen weitaus größeren Zuspruch (bis zu 44 Teilnehmer). Am geringsten war der Zuspruch dagegen in der Innenstadt mit elf interessierten älteren Menschen. Viele der Anwesenden hatten sich bereits bei einer vor den Zukunftswerkstätten liegenden Informationsveranstaltung, bei der die Ergebnisse der Seniorenbefragung vorgestellt wurden, in Interessentenlisten eingetragen. So konnten sie gezielt schriftlich eingeladen werden.

Um die Aufnahmefähigkeit und Mitwirkungsbereitschaft der zu einem großen Teil hoch betagten und körperlich eingeschränkten Teilnehmer nicht über Gebühr zu strapazieren, wurden die Veranstaltungen auf eine Dauer von etwa vier Stunden ausgelegt. Nach der Begrüßung und Einführung in die Veranstaltung, bei der unter anderem der Ablauf und die Gesprächsregeln für die Zusammenarbeit vorgestellt wurden und die Teilnehmer ihre Erwartungen an die Veranstaltungen mitteilen konnten, begann die „Bestandsaufnahme-/Kritikphase“. Unter der Überschrift „Was stört Sie 

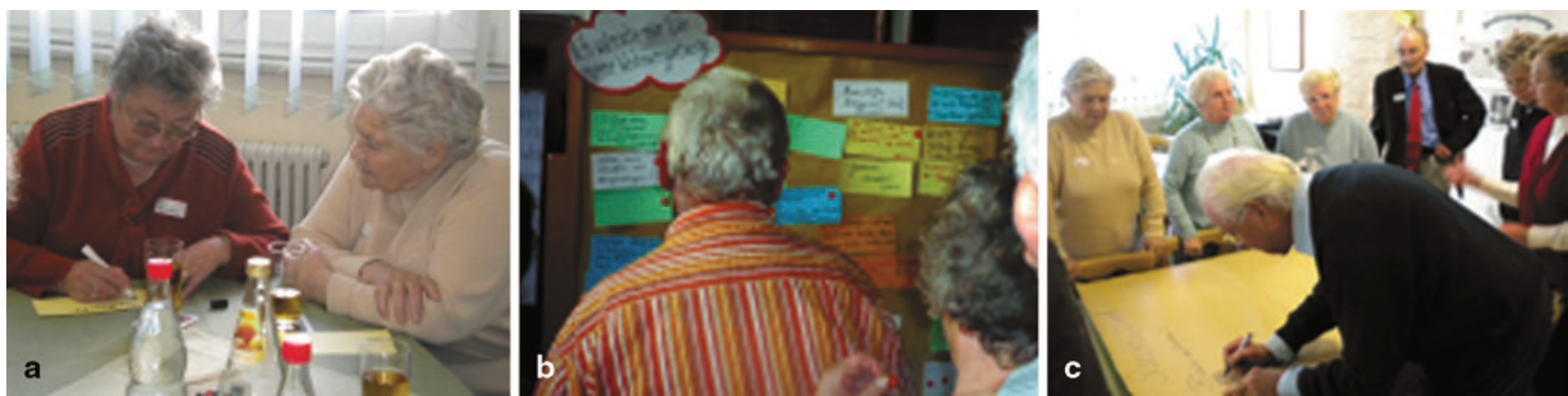

Abb. 2 Ältere Bürger bei den Zukunftswerkstätten. a. Beschriften von Karten mit Ideen b. Identifizierung der wichtigsten Vorschläge c. Konkretisierung der Projekte in der dritten Phase

in Ihrer Wohnumgebung?" konnten die Teilnehmer ihrem Ärger Luft machen über alles, was im Stadtteil nicht ihren Wünschen entsprach. So wurden in sechs Veranstaltungen insgesamt 162 Kritikpunkte gesammelt.

Im Anschluss folgte die „Phantasie-/Utopiephase“. Hier trugen die Anwesenden zu der Frage „Ich wünsche mir für meine Wohnumgebung..." Ideen und Vorschläge für ein ideales Wohngebiet zusammen (vgl. Abb. 2a). Unabhängig von in der Realität bestehenden Einschränkungen hinsichtlich Kosten oder Zuständigkeiten überlegten sich die Teilnehmer Visionen für ihr Wohnumfeld. Im Ganzen waren es 148 Wünsche, die geäußert wurden.

Schließlich wurden in der „Verwirklichungs-/Praxisphase" die entwickelten Ideen von den anwesenden Senioren nach ihrer Dringlichkeit bewertet (vgl. Abb. 2b). Jeweils zwei bis fünf als besonders dringlich identifizierte Ideen wurden anschließend von den Anwesenden auf ihre Durchführbarkeit hin überprüft. Dies geschah anhand der „W-Fragen“ (Was? Wie? Wer? Mit wem? Wann? Wo?) (vgl. Abb. 2c). Auf diese Weise wurden konkrete Projektideen erarbeitet. Die Personen, die sich für die weitere Mitwirkung bei der Umsetzung interessierten, notierten ihre Namen auf den jeweiligen Plakaten aus der dritten Phase.

Einige Teilnehmer waren zunächst sehr zurückhaltend damit, Kritik und Wünsche zu formulieren. Häufig zu hören waren Aussagen wie „Ich bin doch eigentlich mit allem zufrieden“. Diese subjektive Bewertung des Wohnumfeldes stimmt allerdings nicht zwangsläufig mit der Beurteilung durch Außenstehende überein. Besonders bei älteren Menschen lässt sich oft feststellen, dass sie trotz objektiv schlechter Lebensbedingungen zufrieden sind. Dieses Phänomen wird als „Zufriedenheitsparadoxon“ bezeichnet (vgl. Staudinger 2000: 186). Grund hierfür ist ein Kohorteneffekt. Da die älteren Menschen meist entbehrungsreiche Zeiten erlebt haben, erscheint ihnen ihre heutige Situation vergleichsweise vorteilhaft. Diese geringere Anspruchshaltung wird in Zukunft wahrscheinlich seltener anzutreffen sein, da die künftigen Älteren lange andauernde Wohlstandsphasen und einen Wertewandel hin zu Selbstentfaltung erfahren haben (vgl. Witterstätter 2003: 91).

Manche Teilnehmer bezweifelten, ob sich die entwickelten Projektideen nach den Veranstaltungen würden umsetzen lassen, wodurch es einigen Personen schwerfiel, ihrer Phantasie freien Lauf zu lassen. Hier war es die Aufgabe der Moderatorinnen, die Teilnehmer zu motivieren, ihre Wünsche zu formulieren, ohne Berücksichtigung von möglichen einschränkenden Argumenten. Häufig war es dazu notwendig, mit einzelnen Personen gezielt über ihre persönliche Situation zu sprechen, um sie so bei der Formulierung ihrer Anliegen zu unterstützen.

Generell war zu Beginn der Veranstaltungen festzustellen, dass die Teilnehmer erwarteten, es würden bereits fertige Projekte präsentiert. Sie waren überrascht, dass sie selbst gefragt waren, Vorschläge und Ideen einzubringen. Trotz anfänglichen Zögerns war das Feedback am Ende der Veranstaltungen durchweg positiv, denn viele Teilnehmer zeigten sich begeistert davon, nach ihrer Meinung gefragt $\mathrm{zu}$ werden und eigene Vorschläge einbringen zu können. Das strukturierte Vorgehen und die Vorbereitung fanden Zustimmung bei den Älteren, die bislang nur in Ausnahmefällen mit Moderationstechniken und -materialien vertraut waren. Allerdings wurde trotz der allgemein positiven Rückmeldungen auch in den jeweiligen Abschlussrunden bezweifelt, dass die Umsetzung der Projektideen durch die Stadt Pirmasens unterstützt werden würde. Diese pessimistische Einstellung zu Ergebnissen von Zukunftswerkstätten war im Rahmen anderer Projekte mit älteren Menschen nicht spürbar - auch wenn hier teilweise objektiv deutlich schlechtere Ausgangsbedingungen bestanden (vgl. Spellerberg/Woll 2011). ${ }^{3}$

\footnotetext{
${ }^{3}$ In einem ExWoSt-Forschungsprojekt des Landes Rheinland-Pfalz mit dem Titel „Wohneigentum für Generationen“ wurden ebenfalls Zukunftswerkstätten durchgeführt $(\mathrm{n}=5)$. Die Zielgruppen waren Siedlergemeinschaften, die in den 1930er bis 1970er Jahre gegründet wurden, teilweise mit wenigen bis gar keinen Infrastruktureinrichtungen auskommen müssen und ebenfalls ein sehr hohes Durchschnittsalter aufweisen.
} 


\section{Ergebnisse der Zukunftswerkstätten}

Ein wichtiges Anliegen der Senioren, das auf allen sechs Veranstaltungen angesprochen wurde, war die Verkehrssituation und -beruhigung. In diesem Bereich erfolgten insgesamt 30 Nennungen in der ersten Phase, insgesamt 22 Wünsche wurden dazu geäußert und in vier Zukunftswerkstätten wurden Projektideen zu diesem Themenbereich entwickelt. Das zweitwichtigste Thema bildeten die soziale Einbindung im Wohngebiet und das Verhältnis innerhalb der Nachbarschaft. Zwar wurde dahingehend kaum Kritik geübt, dennoch wurden bei drei der sechs Zukunftswerkstätten Wünsche geäußert, die auf die Verbesserung sozialer Netzwerke abzielten (insgesamt 23 Nennungen); in allen drei Fällen wurden Projektideen erarbeitet. Es wurde gewünscht, Kontaktmöglichkeiten und Begegnungsräume für Alteingesessene und Zugezogene, ältere und jüngere Menschen zu schaffen. Ein weiteres dominierendes Thema war die ungenügende Versorgung mit bzw. die schlechte Erreichbarkeit von Einkaufsmöglichkeiten für den täglichen Bedarf und anderen Versorgungseinrichtungen (z. B. Post, Geldinstitut). In der Kritikphase wurden entsprechende Themen 38 Mal benannt, in der Utopiephase 25 Mal. In zwei Fällen entwickelten sich daraus Projektideen. Darüber hinaus wurden bei mehreren Veranstaltungen die Ausgestaltung von Spazier- und Wanderwegen sowie das Aufstellen von Bänken entlang dieser Wege als relevant identifiziert; dies wurde vor allem in der Utopiephase deutlich, wo entsprechende Wünsche insgesamt 13 Mal geäußert worden waren und schließlich in zwei Fällen zur Entwicklung von Projektideen führten. Die Verschmutzung von Straßen und Grünanlagen oder die Gestaltung von Plätzen mit Blumen wurden aufgeführt (29 kritische Nennungen, 12 Wünsche), es entstanden allerdings keine Projektideen. Dies trifft auch auf die Kritik an den Verbindungen und der Ausgestaltung des öffentlichen Nahverkehrs zu (18 kritische Nennungen und elf Wünsche). In diesem Zusammenhang ist auch die mangelnde Barrierefreiheit von Wegen und Straßen in der Wohnumgebung (13 Nennungen in der ersten und fünf in der zweiten Phase) zu erwähnen.

Mit der Umsetzung der Projektideen wurde in den meisten Fällen in den Tagen nach den Veranstaltungen begonnen. Dabei wurden die Engagierten unterstützt durch Mitarbeiter der Stadtverwaltung aus dem Bereich Soziales und dem Seniorenbüro. So konnte sichergestellt werden, dass die Wünsche nicht nur Wünsche bleiben, sondern auch im Rahmen der Möglichkeiten verwirklicht werden.

Die Projekte, die weitere Unterstützung durch Politik und Verwaltung benötigten (etwa der Bau verkehrsberuhigender Maßnahmen), wurden im Seniorenbeirat und im Stadtrat diskutiert und anschließend von den zuständigen Fachstellen weiterbearbeitet. Zur Umsetzung vieler Maßnahmen war darüber hinaus Kommunikation und Kooperation beispielsweise mit den betrauten Fachplanungen, anderen Verwaltungsstellen und politischen Gremien notwendig (vgl. Selle 1996: 61). Einbezogen wurden etwa das Gartenund Friedhofsamt, verschiedene Ortsbeiräte und regionale Einzelhändler.

Nach Rücksprache mit der Stadt Pirmasens zeigte sich etwa ein Jahr nach Abschluss des Projektes, dass nahezu alle Ideen weiterverfolgt und -entwickelt wurden und teilweise bereits abgeschlossen werden konnten. Als Beispiel für eine erfolgreich umgesetzte Idee der Senioren kann das Projekt „Wanderwege“ angeführt werden, das in der Umsetzung breite Aufmerksamkeit und eine rege Beteiligung bei den Anwohnern gefunden hatte. In einer der Zukunftswerkstätten wurde eine Gruppe gebildet, deren Ziel war, gemeinsam mit Mitarbeitern der Stadt Wanderwege aufzuwerten. Im ersten Schritt wurden Spenden für Material gesammelt, anschließend wurden die Sitzbänke in Eigenarbeit aufgestellt. Auch ein Nachbarschaftstreff von Älteren organisierte sich bereits während einer Veranstaltung, der nun regelmäBig stattfindet.

Weitere Ideen können in Zukunft bei der neu eingerichteten „Ehrenamtsbörse“ des Seniorenbüros aufgenommen und gemeinsam mit freiwilligen Engagierten umgesetzt werden. Damit sind über motivierend wirkende Einzelprojekte hinaus dauerhafte Unterstützungsstrukturen geschaffen worden, die gewährleisten, dass ein Teil der Arbeit der Freiwilligen nachhaltigen Einfluss hat. Ziel dieser Einrichtung ist es, neben der Unterstützung bei der Umsetzung von Projekten als Vermittlungsstelle zwischen Angebot und Nachfrage nach freiwilligem Engagement zu fungieren. Ein Schwerpunkt liegt derzeit auf der Vermittlung von freiwilligen „Seniorenbegleitern“ an ältere Menschen, die sich Unterstützung im Alltag wünschen, etwa beim Spazierengehen oder Einkaufen.

\section{Zusammenfassung und Folgerungen}

Ein Vergleich der Ergebnisse der drei angewandten Methoden (repräsentative Befragung, Experteninterviews und Zukunftswerkstätten) zeigt, dass eine ideale Wohnumgebung für die älteren Bürger der Stadt Pirmasens vor allem eine Gestaltung voraussetzt,

- die Mobilität ermöglicht und fördert: Eine selbstbestimmte Gestaltung des Lebens ist in hohem Maße von der Möglichkeit zur Aufrechterhaltung der Mobilität abhängig. Es besteht ein hohes Bedürfnis nach (Freizeit)Mobilität, das aufgrund mangelnder Barrierefreiheit oder Unzufriedenheiten mit dem öffentlichen Nahverkehr häufig nicht erfüllt werden kann. Zusätzlich geht eine Mobilitätsinfrastruktur für ältere Menschen in der Öffentlichkeit mit der Notwendigkeit einher, sich ausru- 
hen zu können. Auch das Fehlen öffentlicher Toiletten weist auf eine nicht ausreichend berücksichtigte Barriere für die Mobilität Älterer hin. Fehlt die Sicherheit, eine qualitativ hochwertige Toilette aufsuchen zu können, wird Mobilität indirekt eingeschränkt.

- die soziale Kontaktnetze in der Nachbarschaft unterstützt: Die Gemeinschaft vor Ort erscheint zentral für die Älteren, denn sie bietet die Möglichkeit, Einsamkeit und Isolation vorzubeugen. Auch das subjektive Sicherheitsgefühl kann sich durch eine starke Gemeinschaft der Bewohner verbessern.

- die Erreichbarkeit insbesondere von Einkaufsmöglichkeiten gewährleistet: Für ältere Menschen, die körperlich und dadurch in ihrer Mobilität eingeschränkt sind bzw. für Personen, die keinen Führerschein besitzen, stellt der Mangel an gut erreichbaren Einkaufsmöglichkeiten im Wohnumfeld eine große Einschränkung dar. Dies hat zusätzlich eine negative Auswirkung auf die sozialen Kontakte: In den Experteninterviews, die im Rahmen des Projektes durchgeführt wurden, wurde festgestellt, dass das Einkaufen für ältere Menschen häufig eine Aktivität ist, die nicht bloß der Beschaffung von lebensnotwendigen Dingen dient, sondern als Form der Kommunikation und Interaktion mit anderen Menschen genutzt wird (vgl. dazu auch Wiswede 2001: 22).

Auch die Sauberkeit des Umfeldes ist vielen Senioren wichtig. Dies weist darauf hin, dass die Wohnqualität nicht nur von Merkmalen der Ausstattung abhängig ist, sondern auch von der symbolischen Wirkung der Wohnumgebung beeinflusst wird.

Der angewandte Methodenmix hat sich als sehr ertragreich erwiesen. Es konnten (1) vertiefte Informationen über die Lebenssituation der Zielgruppe gewonnen und Problemgruppen identifiziert werden, (2) konnten die Sichtweisen unterschiedlicher Multiplikatoren einbezogen werden und (3) mit den Zukunftswerkstätten gezielt betroffene ältere Menschen aktiviert werden. Zukunftswerkstätten haben sich als eine effiziente Methode herausgestellt, um Bedürfnisse, Wünsche und Ideen der Senioren vor Ort in Erfahrung zu bringen und diese direkt in Planungsprozesse der Kommune einzubeziehen. Die Unzufriedenheiten, die in der postalischen Befragung und den Expertengesprächen identifiziert wurden, konnten in den Zukunftswerkstätten wiedergefunden und teilweise bearbeitet werden. Auch das Ergebnis, wonach ein hohes Potenzial an Engagement in der älteren Bevölkerung der Stadt besteht, konnte bestätigt werden. Die in den Zukunftswerkstätten benannten Veränderungswünsche waren aber im Vergleich mit denen aus der Seniorenbefragung wesentlich detaillierter und bezogen sich auf konkrete nahräumliche Gegebenheiten. Die erarbeiteten Projektideen und ihre Realisation zeigen für die Bürger einen deutlicheren, konkreteren Nutzen als die Informationen aus der postalischen Befragung oder den Experteninterviews. Sie aktivieren die älteren Menschen zur Mitwirkung bei der seniorengerechten Gestaltung ihrer Wohnumgebungen. Im gesamten Projekt zeigte sich ein großes Interesse an der Studie und ein großes Potenzial der älteren Generation, sich für die eigenen Belange einzusetzen. Die Abschlussveranstaltung zum Ende der Projektlaufzeit, bei der die Ergebnisse der Untersuchungsschritte sowie erste Umsetzungserfolge der erarbeiteten Projektideen präsentiert wurden, stieß mit einer Teilnehmerzahl von etwa 70 Personen auf große Resonanz bei der älteren Generation.

Angesichts der oft schwierigen finanziellen Lage der Kommunen können Zukunftswerkstätten in der beschriebenen Form ein Mittel sein, Gestaltungsmöglichkeiten zu erhalten und zu erweitern, auch wenn diese selbst mit Kosten verbunden sind. Zukunftswerkstätten bieten einige Vorteile: Einerseits wird den Betroffenen gezeigt, dass ihre Probleme ernst genommen werden, wodurch die Zufriedenheit und auch die Identifikation der Bürger mit ihrer Stadt erhöht werden kann. Andererseits können Informationen ermittelt werden über die Situation und Bedürfnisse der Betroffenen und es kann gemeinsam mit ihnen über Lösungswege diskutiert werden. Vorhandene Kompetenzen und Kenntnisse vor Ort werden genutzt. Es entsteht außerdem Motivation zur Eigeninitiative, was langfristiges unentgeltliches und produktives Engagement hervorbringt, das an den Bedürfnissen der Betroffenen ansetzt und damit zu einer höheren Lebensqualität beiträgt.

Es ist als besonderer Vorteil der durchgeführten Zukunftswerkstätten zu bewerten, dass sie in ihrem Verlauf zeitlich stark komprimiert und kompakt waren, trotzdem aber wesentliche Punkte zur Sprache kamen. Das stringente und zugleich abwechslungsreiche Verfahren stellten die Senioren selbst als besonders positiv heraus. Hierzu trugen auch der flexible Umgang mit Pausen und deren Gestaltung bei: Jeweils etwa nach der Hälfte des Ablaufs einer Veranstaltung wurde eine kurze Pause eingelegt, in der Kaffee und Kuchen angeboten wurden. Dies stärkte die Zufriedenheit und Motivation der Teilnehmer und brachte eine positive und ungezwungene Gesamtatmosphäre mit sich. Es ist wichtig einzubeziehen, dass Engagement und freiwillige Arbeit den Beteiligten Freude machen sollen (vgl. dazu auch Gensicke/Picot/Geiss 2006: 289).

Kritisch ist anzumerken, dass es sich bei den Teilnehmern der Zukunftswerkstätten nicht um eine repräsentative Auswahl älterer Menschen handelte. Die Ergebnisse spiegeln nicht die Meinung der älteren Pirmasenser Bevölkerung insgesamt wider, sondern sie sind als selektiv zu betrachten. Gerade die Personengruppe, für die in erster Linie eine Verbesserung erzielt werden soll, hat womöglich besonders hohe Hürden zu überwinden, ihre Problemsicht einzubringen. Durch kurze Wege innerhalb der einzelnen Stadtgebiete und das Engagement von Mitarbeitern der Stadt Pirmasens, 
die einen Abholservice für die mobilitätseingeschränkten Personen anboten, konnte diese Schwierigkeit im beschriebenen Projekt gemindert werden.

Zudem ist darauf hinzuweisen, dass es sich bei Zukunftswerkstätten um einen lokalen, stadtteilbezogenen „Bottom-up"-Prozess handelt, der quer liegen kann zu den bestehenden Strukturen und Strategien der Stadt. Die Umsetzung der daraus entstandenen Ideen verlangt Energien an Stellen, die möglicherweise nicht eingeplant waren. Fürst/Scholles (2008) beschreiben, dass Partizipation oft als „Störfaktor“ in etablierten Systemen angesehen wird: „die tradierten Entscheidungsstrukturen werden dadurch aufgebrochen, im vorparlamentarischen Raum ausgehandelte Ergebnisse werden wieder infrage gestellt, zusätzliche mitsprechende Akteure erfordern immer höheren Einsatz an Zeit, Personal und Finanzen, und generell wehren sich Institutionen gegen externe Impulse, die sie schlecht kontrollieren können“" (Fürst/Scholles 2008: 163). Bei der Planung seniorengerechter Wohngebiete bedarf es aber ständiger Weiterentwicklung, um auf die sich wandelnden Bedürfnisse eingehen zu können. Der Dialog, insbesondere mit den Personen, die ihr Interesse an der Umsetzung der Projektvorschläge bekundet haben, setzt neue Potenziale frei. Im Prozess ist es notwendig, kontinuierlich zu prüfen, welche auf den Zukunftswerkstätten beschlossenen Maßnahmen schon erfolgt sind und welche weiteren Schritte notwendig sind. Darüber hinaus erscheint die Vernetzung mit Akteuren aus Vereinen, lokalen Betrieben und anderen Organisationen für die Umsetzung der Projektideen wichtig. Dauerhaft werden Strukturen benötigt, die eine kontinuierliche Zusammenarbeit an den Projektideen ermöglichen.

Den Kommunen kommt eine zentrale Bedeutung für die Anregung und Unterstützung des freiwilligen Engagements zu, etwa durch gezielte Information, Beratung, Förderung und Vermittlung. Für die Gewinnung weiterer Freiwilliger zur Unterstützung von Projekten ist eine geeignete Strategie der Ansprache unerlässlich. ${ }^{4}$ Eine einseitige ,Verpflichtung“ der Bürger mit dem Ziel, das Engagement der Älteren als Ersatz für Leistungen heranzuziehen, die von kommunaler oder privater Seite nicht mehr erbracht werden (können), wird dabei kaum funktionieren. Vielmehr sind die Kommunen aufgefordert, Strukturen zu schaffen und zu finanzieren, die es ermöglichen, den bürgerschaftlichen Einsatz zu unterstützen (vgl. Deutscher Verein für öffentliche und private Fürsorge 2006: 23 ff.). Viele Ältere sind bereit sich einzubringen, sie müssen aber auf eine Weise motiviert und

\footnotetext{
${ }^{4}$ Wie schwer dies ist, zeigte sich auch in diesem Projekt. Die Ansprache zur Gründung der Ehrenamtsbörse im Seniorenbüro der Stadt erzielte zunächst nicht die gewünschte Wirkung, die Anwesenden zum Mitmachen zu motivieren, da in ihr nicht vermittelt wurde, welchen persönlichen und gesellschaftlichen Gewinn eine freiwillige Tätigkeit bedeuten kann.
}

auch anerkannt werden, die positiv in ihrer alltäglichen Lebenswelt wirkt.

Danksagung Die Autorinnen bedanken sich herzlich bei der Stadt Pirmasens für die Förderung der Studie, die Bereitstellung von Informationen und die fortlaufende konstruktive Kooperation.

\section{Literatur}

Banse, J.; Möbius, M.; Deilmann, C. (2008): Wohnen im Alter 60+. Ergebnisse einer Befragung in der Stadt Dresden. Dresden. = IÖR-Texte, Nr. 156.

Bischoff, A.; Selle, K.; Sinning, H. (2007): Informieren, Beteiligen, Kooperieren. Kommunikation in Planungsprozessen. Eine Übersicht zu Formen, Verfahren und Methoden. Dortmund.

Brauers, S. (2008): Potenziale der Älteren in Kommunen nutzen. Das Bundesmodellprogramm „Erfahrungswissen für Initiativen“ im europäischen Kontext. In: Erlinghagen, M.; Hank, K (Hrsg.): Produktives Altern und informelle Arbeit in modernen Gesellschaften. Wiesbaden, 215-234. = Alter(n) und Gesellschaft, Band 16.

Braun, J.; Bischoff, S. (1999): Bürgerliches Engagement älterer Menschen: Motive und Aktivitäten. Engagementförderung in Kommunen - Paradigmenwechsel in der offenen Altenarbeit. Stuttgart, Berlin, Köln. = Schriftenreihe des Bundesministeriums für Familie, Senioren, Frauen und Jugend, Band 184.

Bundesinstitut für Bau-, Stadt- und Raumforschung (BBSR) (2010): INKAR 2009 - Indikatoren, Karten und Graphiken zur Raumund Stadtentwicklung in Deutschland und in Europa. CD-ROM. Bonn.

Bundesministerium für Familie, Senioren, Frauen und Jugend (BMFSFJ) (2010): Sechster Bericht zur Lage der älteren Generation. Altersbilder in der Gesellschaft. Berlin.

Deutscher Bundestag (2002): Bericht der Enquete-Kommission „Zukunft des Bürgerschaftlichen Engagements“. Bürgerschaftliches Engagement: auf dem Weg in eine zukunftsfähige Bürgergesellschaft. Bundestags-Drucksache 14/8900. Berlin.

Deutscher Verein für öffentliche und private Fürsorge (2006): Empfehlungen zur Gestaltung der sozialen Infrastruktur in den Kommunen mit einer älter werdenden Bevölkerung. Online unter http://www.deutscher-verein.de/05-empfehlungen/empfehlungen_archiv/empfehlungen2006/pdf/copy8_of_eugleichbehandlungsrichtlinien.pdf (letzter Zugriff am 03.03.2011).

Engstler, H.; Menning, S.; Hoffmann, E.; Tesch-Römer, C. (2004): Die Zeitverwendung älterer Menschen. In: Statistisches Bundesamt (Hrsg.): Alltag in Deutschland. Analysen zur Zeitverwendung. Stuttgart, 216-246. = Forum der Bundesstatistik, Band 43.

Erlinghagen, M.; Hank, K. (Hrsg.) (2008): Produktives Altern und informelle Arbeit in modernen Gesellschaften. Wiesbaden. = Alter(n) und Gesellschaft, Band 16.

Friedrichs, J. (1990): Aktionsräume von Stadtteilbewohnern verschiedener Lebensphasen. In: Bertels, L.; Herlyn, U. (Hrsg.): Lebenslauf und Raumerfahrung. Opladen, 161-178.

Fürst, D.; Scholles, F. (2008): Partizipative Planung. In: Fürst, D.; Scholles, F. (Hrsg.): Handbuch Theorien und Methoden der Raum- und Umweltplanung. Dortmund, 161-178.

Gensicke, T. (2010): Zivilgesellschaft und freiwilliges Engagement in Deutschland im Lichte des Freiwilligensurveys 1999, 2004 und 2009. In: Zeitschrift für Erziehungswissenschaft 13, 2, 215-232.

Gensicke, T.; Picot, S.; Geiss, S. (2006): Freiwilliges Engagement in Deutschland 1999 - 2004. Ergebnisse der repräsentativen Trenderhebung zu Ehrenamt, Freiwilligenarbeit und bürgerschaftlichem Engagement. Wiesbaden. 
Haski-Leventhal, D. (2009): Elderly Volunteering and Well-Being: A Cross-European Comparison Based on SHARE Data. In: Volun$\operatorname{tas} 20,4,388-404$

Heetderks, G.; Nell, K. (2008): Für, mit und von Ältere(n). Neue Rollenprofie von hauptamtlich und freiwillig Tätigen in der sozialen und kulturellen Arbeit. In: Sozial Extra 32, 5-6, 22-25.

Höpflinger, F. (2004): Traditionelles und neues Wohnen im Alter. Age Report 2004. Zürich.

Jungk, R.; Müllert, N. R. (1997): Zukunftswerkstätten. Mit Phantasie gegen Routine und Resignation. München.

Karl, F.; Aner, K.; Bettmer, F.; Olbermann, E. (2008): Perspektiven einer neuen Engagementkultur. Praxisbuch zur kooperativen Entwicklung von Projekten. Wiesbaden.

Kuhnt, B.; Müllert, N. R. (2006): Moderationsfibel Zukunftswerkstätten - verstehen - anleiten - einsetzen. Neu-Ulm.

Müllert, N. R. (2009): Zukunftswerkstätten. Über Chancen demokratischer Zukunftsgestaltung. In: Popp, R.; Schüll, E (Hrsg.): Zukunftsforschung und Zukunftsgestaltung. Beiträge aus Wissenschaft und Praxis. Berlin, Heidelberg, 269-276.

Petermann, H.; Roth, M. (2006): Alter: Produktiver Umgang mit den Aufgaben einer Lebensphase. In: Renneberg, B.; Hammelstein, P (Hrsg.): Gesundheitspsychologie. Heidelberg, 245-264.

Pfenning, U.; Benighaus, C. (2008): Partizipativer Wandel - methodischer Wandel: Neue und klassische Formen der Bürgerbeteiligung im Vergleich. In: Vetter, A. (Hrsg.): Erfolgsbedingungen lokaler Bürgerbeteiligung. Wiesbaden, 195-216. = Städte und Regionen in Europa, Band 16.

Rohden, K. S.; Villard, H. J. (2010): Kommunale Alten(hilfe-)planung - Rahmung und Standards. In: Aner, K.; Karl, U (Hrsg.): Handbuch Soziale Arbeit und Alter. Wiesbaden, 51-57.

Rosenmayr, L. (1996): Altern im Lebenslauf. Soziale Position, Konflikte und Liebe in den späten Jahren. Göttingen.

Schmidt, R. (2004): Förderung der Potenziale im Alter als Zukunftsthema der Politik. In: Sozialer Fortschritt 53, 11-12, 326-328.
Schroeter, K. R.; Zängl, P. (Hrsg.) (2006): Altern und bürgerschaftliches Engagement. Aspekte der Vergemeinschaftung und Vergesellschaftung in der Lebensphase Alter. Wiesbaden. = Alter(n) und Gesellschaft, Band 12.

Selle, K. (1996): Von der Bürgerbeteiligung zur Kooperation und zurück. In: Selle, K. (Hrsg.): Planung und Kommunikation. Gestaltung von Planungsprozessen in Quartier, Stadt und Landschaft. Grundlagen, Methoden und Praxiserfahrungen. Wiesbaden, Berlin, 61-78.

Sellnow, R. (1998): Die Methode der Zukunftswerkstatt. In: Braczyk, H. J.; Kerst, C.; Seltz, R (Hrsg.): Kreativität als Chance für den Standort Deutschland. Berlin u. a., 145-152.

Spellerberg, A.; Krickel, P. (2010): Planung für Senioren in Pirmasens. Empirische Studien und sozialwissenschaftliche Begleitung. Kaiserslautern. Online unter: http://d-nb.info/1001097254/34/ (letzter Zugriff am 03.03.2011).

Spellerberg, A.; Woll, T. (2011): Wohneigentum im Wandel. Siedlergemeinschaften gestalten ihre Zukunft. Kaiserslautern (unveröffentlichtes Manuskript).

Statistisches Bundesamt (2009): Bevölkerung Deutschlands bis 2060. 12. koordinierte Bevölkerungsvorausberechnung. Wiesbaden.

Staudinger, U. M. (2000): Viele Gründe sprechen dagegen, und trotzdem geht es vielen Menschen gut: Das Paradox des subjektiven Wohlbefindens. In: Psychologische Rundschau 51, 4, 185-197.

Thabe, S. (1997): Alte Menschen im Stadtteil. Handlungsansätze für soziale und kulturelle Einrichtungen. Dortmund.

Wiswede, G. (2001): Senioren in Deutschland: ihre Lebenssituation und daraus resultierende Einflüsse auf Freizeit-, Konsum- und Ernährungsverhalten. In: Leonhäuser, I. U.; Berg, I (Hrsg.): Lebensstile und Alter. 14. Symposium Wissenschaft und Ernährungspraxis. Bingen, 10-27.

Witterstätter, K. (2003): Soziologie für die Altenarbeit - Soziale Gerontologie. Freiburg im Breisgau. 